\title{
Automated nasopharyngeal carcinoma segmentation in magnetic resonance images by combination of convolutional neural networks and graph cut
}

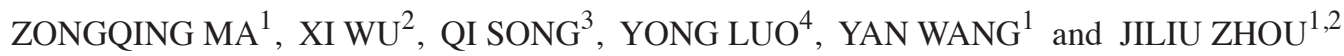 \\ ${ }^{1}$ College of Computer Science, Sichuan University, Chengdu, Sichuan 610065; ${ }^{2}$ School of Computer Science, \\ Chengdu University of Information Technology, Chengdu, Sichuan 610225, P.R. China; ${ }^{3}$ CuraCloud Corp., \\ Seattle, WA 98104, USA; ${ }^{4}$ Department of Head and Neck and Mammary Oncology, \\ West China Hospital, Sichuan University, Chengdu, Sichuan 610041, P.R. China
}

Received December 1, 2017; Accepted June 22, 2018

DOI: $10.3892 /$ etm.2018.6478

\begin{abstract}
Accurate and reliable segmentation of nasopharyngeal carcinoma (NPC) in medical images is an import task for clinical applications, including radiotherapy. However, NPC features large variations in lesion size and shape, as well as inhomogeneous intensities within the tumor and similar intensity to that of nearby tissues, making its segmentation a challenging task. The present study proposes a novel automated NPC segmentation method in magnetic resonance (MR) images by combining a deep convolutional neural network (CNN) model and a 3-dimensional (3D) graph cut-based method in a two-stage manner. First, a multi-view deep CNN-based segmentation method is performed. A voxel-wise initial segmentation is generated by integrating the inferential classification information of three trained single-view CNNs. Instead of directly using the CNN classification results to achieve a final segmentation, the proposed method uses a 3D graph cut-based method to refine the initial segmentation. Specifically, the probability response map obtained using the multi-view CNN method is utilized to calculate the region cost, which represents the likelihood of a voxel being assigned to the tumor or non-tumor. Structure information in $3 \mathrm{D}$ from the original MR images is used to calculate the boundary cost, which measures the difference between the two voxels in the 3D neighborhood. The proposed method was evaluated on T1-weighted images from 30 NPC patients using the leave-one-out method. The experimental results demonstrated that the proposed method is effective and accurate for NPC segmentation.
\end{abstract}

Correspondence to: Professor Jiliu Zhou, College of Computer Science, Sichuan University, 24 South Section 1 of Yihuan Road, Chengdu, Sichuan 610065, P.R. China

E-mail: zhoujiliu@cuit.edu.cn

Key words: nasopharyngeal carcinoma, image segmentation, convolutional neural networks, graph cut, magnetic resonance imaging

\section{Introduction}

Nasopharyngeal carcinoma (NPC) is a type of head and neck cancer that originates in the nasopharynx, and has a high incidence in South China, Southeast Asia, the Arctic, the Middle East and North Africa (1). As a malignant tumor type, NPC seriously threatens the health and life of humans. Magnetic resonance imaging (MRI) has been utilized as a preferred imaging modality for the evaluation of local, regional and intracranial infiltration of NPC in clinical practice, due to its high spatial resolution for examining soft tissues. Accurate delineation of NPC has an important role not only for radiotherapy planning, but also for follow-up evaluations. At present, nasopharyngeal tumor borders are drawn by slice in clinical practice. However, manual segmentation is tedious, time-consuming and prone to errors. In addition, accurate segmentation highly depends on the operator's expertise and experience. Therefore, semi-automated or automated methods are required to accelerate and facilitate clinical applications. However, this is a challenging task, since nasopharyngeal tumors have a more complex structure and anatomical location than other tumor types, e.g., lung cancer. NPC is spatially close to several tissue types, including bones, muscle and mucosa, which possess similar intensities on imaging, and exhibits significant variations in size and shape among NPC patients. In addition, MRI may have certain limitations, including inhomogeneous intensity or different intensity ranges among the same sequences and acquisition scanners. Fig. 1 presents an annotated slice of an NPC patient's MR image in the axial view. In this image, the tumor area has a similar intensity to that of the surrounding area. Furthermore, the tumor has a highly irregular shape and an infiltrative growth pattern. The present study focuses on automated NPC segmentation of MR images.

Only a small number of studies on semi-automated or automated NPC segmentation are available, likely due to the segmentation challenge and the low incidence of NPC in most countries. Current NPC segmentation approaches may be categorized into three groups: intensity-based, shape-based and statistical approaches. 
Intensity-based methods mainly utilize image intensities. Lee et al (2) introduced an algorithm including image masking, Bayesian probability calculation and seed growing for NPC segmentation. The algorithm was required to be initiated by the user and was evaluated on contrast-enhanced T1-weighted imaging (T1WI) and T2WI. In another, separate study, the initial seed for region growing was generated from probabilistic maps based on prior knowledge of location, intensities and non-tumor regions, forming an automated NPC segmentation framework of CT images (3). In this method, only one seed is selected, which may not be the optimal one. A modified region growing method with localization of computed tomography (CT) images for NPC segmentation was introduced in another study (4), wherein two seeds were asynchronously generated for each testing image, and the first seed relied on the corresponding standard ground truth (GT) images. Since NPC has a similar intensity to that of nearby tissues, intensity-based segmentation methods may have a limited performance.

For the shape-based method, information on size, contour and shape are utilized. Huang et al (5) proposed an automated NPC segmentation method in MR images. In the method, the adaptive nasopharyngeal region location was first calculated, and the contour of the tumor was then estimated by distance regularized level set evolution. A hidden Markov random field (MRF) model with maximum entropy was further utilized to refine the segmentation results. Fitton et al (6) presented a semi-automated delineation for radiotherapy of NPC and user-driven delineation assisted by a snake algorithm was applied on weighted CT-MR images. The algorithm reduced the delineation time, but did not significantly improve the accuracy. In general, the shape-based method is suitable for segmentation of targets with a uniform size, contour or shape, which limits the application in NPC segmentation tasks to a certain extent, as NPCs feature high variations in shape and size.

Regarding statistical approaches, Zhou et al (7) introduced a two-class support vector machine (SVM) for NPC segmentation in MR images, wherein tumor and non-tumor images were projected onto the feature space that was initially created, and the projected vectors were then classified by the SVM. Furthermore, Zhou et al (8) proposed a knowledge-based fuzzy clustering method for MR images. This method implicitly imposed certain prior assumptions on the data distribution. However, it is difficult to predict the data distribution for clinical MR data. Based on these studies, the accuracy and robustness of automated NPC segmentation methods requires improvement.

In recent years, deep convolutional neural networks (CNNs) $(9,10)$, one type of deep learning model, have been popular in the field of medical image segmentation. Contrary to traditional machine learning methods, CNNs do not require a set of hand-crafted features for classification, but automatically learn hierarchies of complex features directly from the raw input. Several studies have applied CNNs to the segmentation of knee cartilage (11), pancreas (12), retinal blood vessels (13) and brain images $(14,15)$. Furthermore, the application of CNNs has also been investigated for tumor segmentation. For instance, Pereira et al (16) explored deep small kernel architecture for segmentation of gliomas in MR images. A shallow CNN with two convolutional layers separated by max-pooling, followed by one fully connected layer and a Softmax layer was

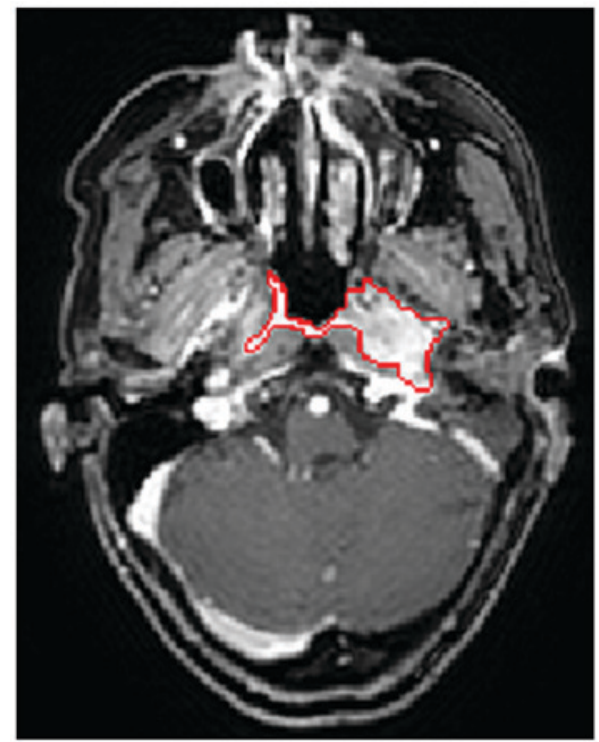

Figure 1. Axial view magnetic resonance imaging slice with manual segmentation of the nasopharyngeal carcinoma. Intensities within the tumor may vary, and the tumor may invade nearby tissue exhibiting similar image intensities.

employed to segment brain tumor tissues (17). Havaei et al (18) developed a two-pathway architecture and a two-stage training for brain tumor segmentation. A post-processing method based on connected components was used to remove flat blobs that may appear in the predictions.

Motivated by the superior performance achieved by deep CNNs in the field of medical image segmentation, the present study proposes a two-stage coarse-to-fine framework unifying a deep CNN and graph cut method for NPC segmentation of MR images. Specifically, a multi-view deep CNN-based segmentation method is proposed in the first stage. It directly learns hierarchical features from MR images and utilizes 3-dimensional (3D) context information via multi-view fusion. A voxel-wise initial segmentation and a probability response map are subsequently generated. A 3D graph cut-based segmentation method is proposed to refine the segmentation results in the second stage. The probability response map obtained from the multi-view CNN method is utilized to calculate the region cost and the structure information of the original MR images is used to calculate the boundary cost. A preliminary version of the current study was first described in a conference paper (19). The present study consolidated the previous study and improved the method. The proposed method is an early attempt of employing a deep CNN model and graph cut method for NPC segmentation, and is a fully-automated approach without any user intervention.

\section{Materials and methods}

Materials and data acquisition. In the present exploration study, T1W images acquired from 30 subjects were used. The age of the patients ranged from 21 to 76 years with a mean age of 50.2 \pm 13.9 years, and the cohort comprised different cancer stages. The T1W images were acquired with a Philips Achieva 3T scanner (Philips, Eindhoven, The Netherlands). The images have the same dimension of 528x528x290 and the same voxel size of $0.61 \times 0.61 \times 0.8 \mathrm{~mm}^{3}$, ranging from the top of the head to 


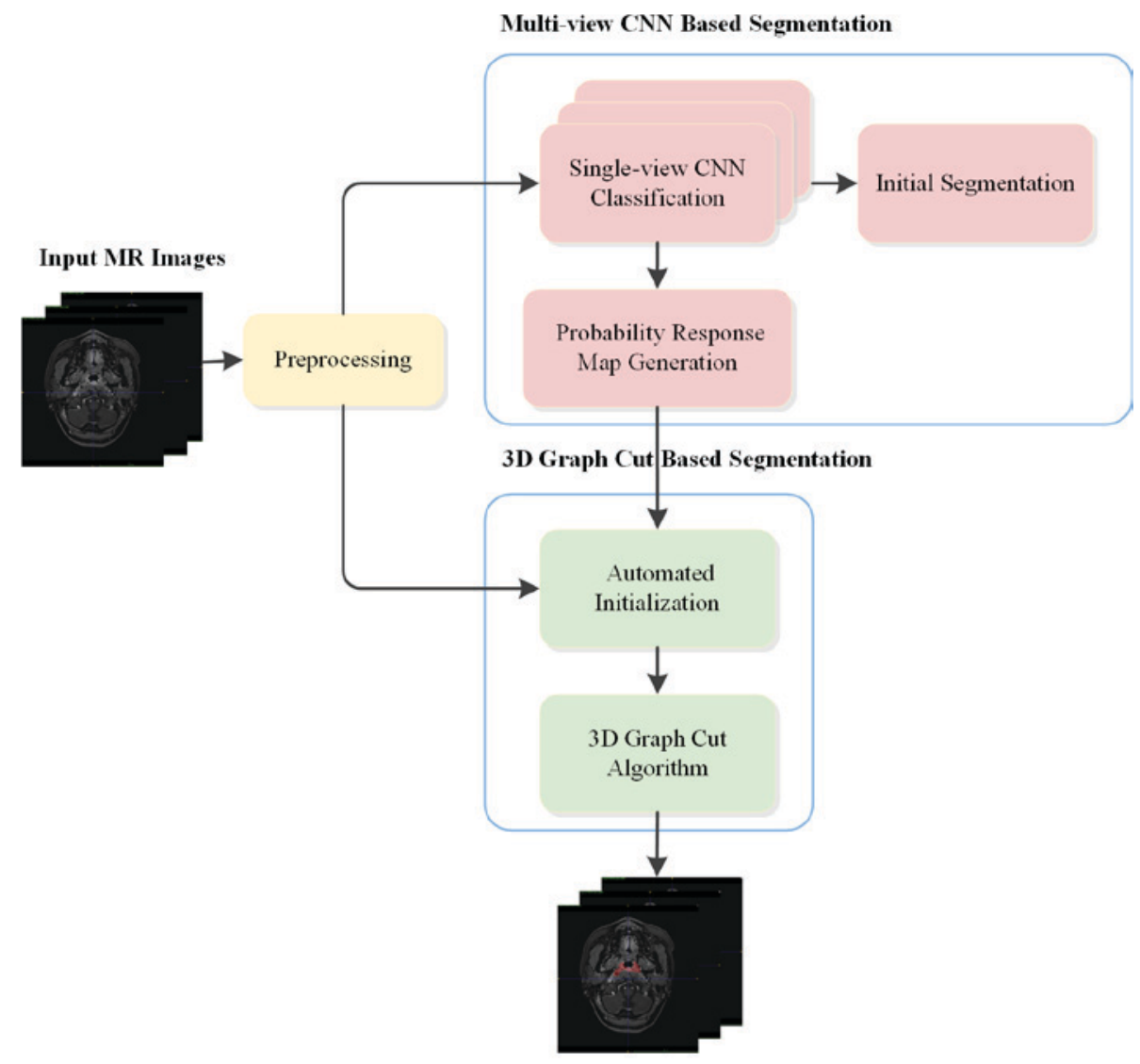

Final Segmentation

Figure 2. Overview of the proposed method. MR, magnetic resonance; 3D, 3-dimensional; CNN, convolutional neural network.

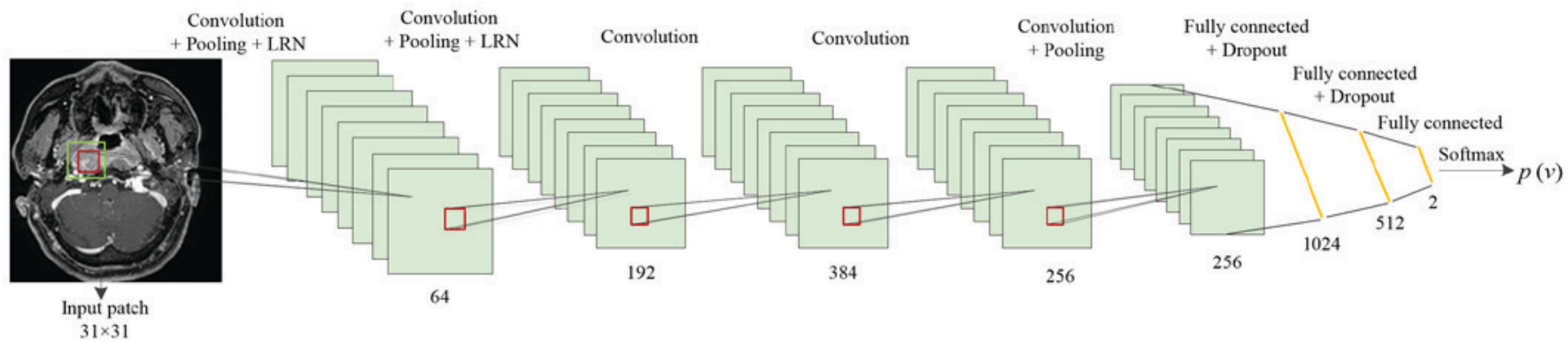

Figure 3. Detailed architecture of the used convolutional neural network in the present study. LRN, local response normalization.

the neck. Manual segmentation of the nasopharyngeal tumor was performed by an experienced radiation oncologist, which was referred as GT. The nasopharyngeal tumor was contoured for all subjects, which was performed slice by slice (in the axial view). Fig. 2 presents an overview of the proposed method, which is described in detail below.

Image pre-processing. Since the MR image is altered by the bias field distortion, the Nick's N3 insight segmentation and registration toolkit implementation for MRI method (20) was applied to each acquired T1W image for bias correction. The intensity normalization method proposed by Nyúl et al (21) was then employed to adjust the contrast and intensity range to a similar level among different patients. Isotropic resampling was subsequently performed on each image to achieve a resolution of $1.0 \times 1.0 \times 1.0 \mathrm{~mm}^{3}$. Considering the acquired images include a large scan volume ranging from head to neck and the nasopharyngeal tumor only occupies a small region, to reduce the computational complexity, the nasopharyngeal region of each image that contained the nasopharyngeal tumor was selected as the volume of interest in the present study.

Multi-view convolutional neural network-based segmentation. As in other studies (14-17), the NPC segmentation problem is considered as a binary classification problem, which means that each voxel in the image is classified as a tumor or non-tumor type. According to the typical CNN architecture for classification, an AlexNet-like CNN architecture was used for the present NPC segmentation (10). The detailed architecture employed is presented in Fig. 3. The network takes image 
patches as input and consists of eight learned layers, namely 5 convolutional layers and 3 fully connected layers. In order to allow the network to learn features that are spatially invariant with respect to the location and make the representation more compact, a max pooling layer was used after the first, second and last convolutional layers. Following the first two max pooling layers, a local response normalization scheme was applied. To perform NPC classification, the CNN architecture ends with a 2-way Softmax layer that computes the following function:

$$
\mathrm{P}(\mathrm{y} \mid \mathrm{I})=\frac{\exp \left(\mathrm{a}_{\mathrm{y}}\right)}{\sum_{\mathrm{k} \in\{0,1\}} \exp \left(\mathrm{a}_{\mathrm{k}}\right)}
$$

where $\mathrm{P}(\mathrm{y} \mid \mathrm{I})$ denotes the probability of patch I being tumor or non-tumor, with $\mathrm{y}=0$ and $\mathrm{y}=1$ indicating non-tumor and tumor respectively. $\mathrm{a}_{\mathrm{y}}$ is each input value to the layer. The output of this layer ranges from 0 to 1 , which may be interpreted as the likelihood of the center voxel being classified as tumor or non-tumor.

The number of feature maps contained in the 5 cascaded convolutional layers was designed to 64, 192, 384, 256 and 256, respectively, and the corresponding filter size was set to $3 \times 3$. A zero padding strategy and a stride size of one voxel were used for all convolutional layers. For all pooling layers, a filter size of $3 \times 3$ and a stride size of $2 \times 2$ were used to retain extensive information regarding location. In addition, the output number of the three fully connected layers was set to 1,024, 512 and 2, respectively.

In order to avoid overfitting, Dropout was used to constrain the first two fully connected layers of the CNN (22). In the training stage, Dropout removes nodes from the network with a probability of 0.5 . In this manner, all nodes of the fully connected layer are required to learn more robust features and reduce complex co-adaptations with each other. In the testing stage, all nodes are used. The activation function is responsible for non-linear transformation of the data. Rectifier linear units, defined as $\mathrm{f}(\mathrm{x})=\max (0, \mathrm{x})$, were identified to expedite the training $(10,23)$ and achieved better results than the classical sigmoid or hyperbolic tangent functions. Thus, rectifier linear units are used as the activation function for each convolutional layer and fully connected layer of the CNN in the present model.

For patch-based CNN classification, the information used to infer the class of each voxel in the image is provided in the form of image patches centered at the target voxel, and certain size patches were extracted from a specified view perpendicular to an axis, which may limit the receptive field of the network. To obtain $3 \mathrm{D}$ context information and considering computation efficiency and memory consumption, the information of patches from all of the three orthogonal views (coronal, sagittal and axial) was integrated to classify the target voxel, providing a multi-view CNN method for NPC segmentation. Specifically, three deep single-view CNNs use the same architecture as aforementioned but are trained separately using patches extracted from the three orthogonal views. Each of them is learned to classify the same target voxel but from a different perspective (axial, sagittal or coronal). Once the training process is completed, the three-way trained single-view CNNs are applied to perform NPC classification and output the likelihood of each voxel to have a tumor or non-tumor identity, respectively. This inferential classification information is then merged by thresholding and determining the

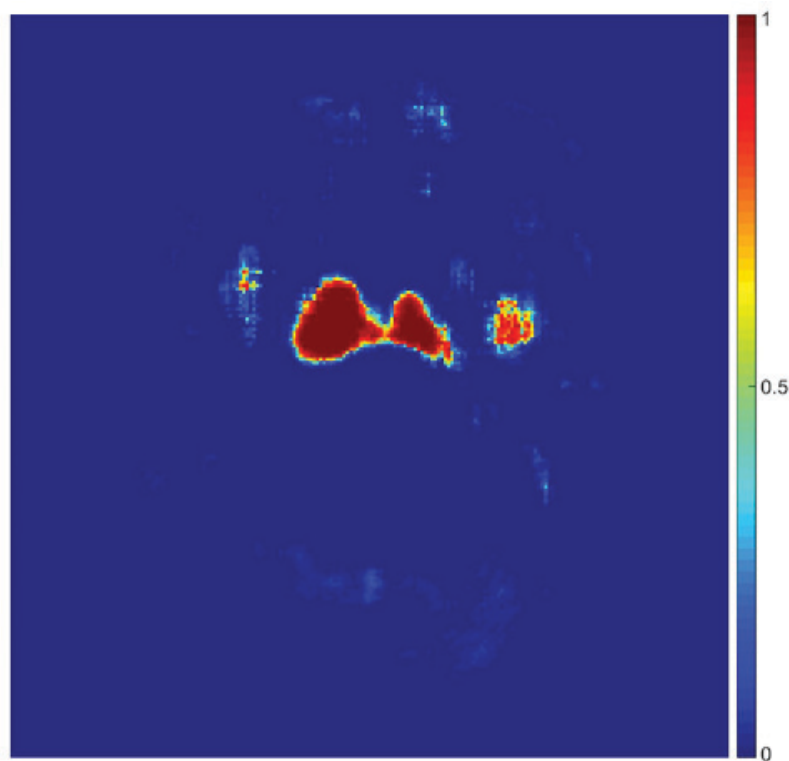

Figure 4. Example slice of the probability response map in a magnetic resonance image of nasopharyngeal carcinoma. A larger value indicates that the voxel has an increased probability to be associated with the tumor.

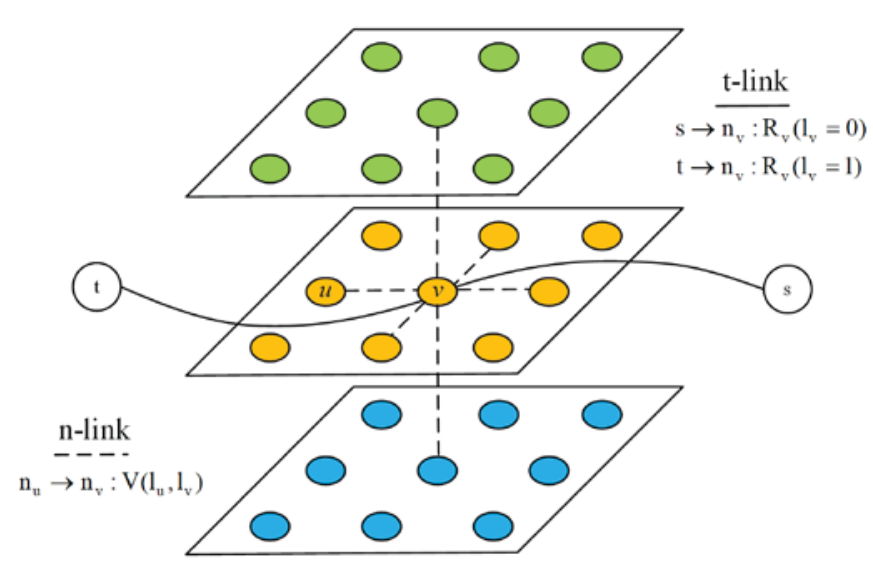

Figure 5. Illustration of 3-dimensional graph construction of the magnetic resonance image segmentation for nasopharyngeal carcinoma. Two types of edges are introduced. The t-link edges (solid lines) and n-link edges (dotted lines) encode the region cost and boundary cost, respectively. s, source node; $\mathrm{t}$, sink node; $\mathrm{u}$ and $\mathrm{v}$, two neighboring voxels in the magnetic resonance image; $n_{u}$ and $n_{v}$, the graph nodes corresponding to $u$ and $v ; 1_{v}$, the binary label assigned to the voxel $v ; R\left(1_{v}\right)$, the region cost assigned to the voxel $v ; V\left(1_{u}, 1_{v}\right)$, the boundary cost between the two neighboring voxels $\mathrm{u}$ and $\mathrm{v}$.

statistical majority to generate the initial segmentation result. Furthermore, the corresponding probability response map, which provides the likelihood of each voxel to be assigned to the tumor, is generated by averaging the class probabilities (p) inferred by the three networks according to the following formula:

$$
\mathrm{p}(\mathrm{l}=1 \mid \mathrm{v})=\frac{1}{3} \sum_{\mathrm{i}=1}^{3} \mathrm{p}_{\mathrm{i}}\left(\mathrm{l}=1 \mid \mathrm{v}, \mathrm{w}_{\mathrm{i}}, \mathrm{b}_{\mathrm{i}}\right)
$$

where 1 denotes the binary label assigned to the voxel $\mathrm{v}$ with $l=1$ meaning that the voxel is assigned to the tumor, and $w$ and $b$ stand for the weight and bias of the CNN network, respectively. An example slice of the probability response map is presented in Fig. 4, which is used to provide prior knowledge in the second stage of the proposed method. 
$3 D$ graph cut-based segmentation. Although the above proposed multi-view CNN method for NPC segmentation is able to capture certain 3D structure information based on the receptive field from convolution, final voxel inferences are independent of each other without the consideration of structural information, and thus, the solution is not globally optimal. To solve this problem, the approach with an MRF solved by graph cut was utilized to refine the segmentation. A graph cut algorithm interprets an image as a graph and formulates the image segmentation problem as an energy minimization problem. The energy cost function is defined according to the application (24-30). Therefore, the graph cut is regarded as a versatile method. In the second stage of the present method, a 3D graph cut based method was used for NPC segmentation.

An MR image I of $\mathrm{N}$ voxels is considered. The associated $3 \mathrm{D}$ graph is formed by $\mathrm{N}$ nodes corresponding to each voxel of the image, plus two additional nodes known as terminal nodes. The source $s$ represents the tumor and the sink t represents the background. Each node at position $n$ in the MR image is connected to its 6 neighbors forming a 3D neighborhood system. These neighborhood edges or links are called n-links and each one of them is weighted by the boundary cost. In addition, each node in the MR image is connected to the terminal nodes $\mathrm{s}$ and $\mathrm{t}$, and these edges are referred to as t-links and are weighted by the region cost. Fig. 5 presents the constructed 3D graph.

The energy cost function for NPC segmentation consist of region terms and boundary terms as in the well-known binary graph cut method (25). It was formulated as follows:

$$
\mathrm{E}(\mathrm{l})=\sum_{\mathrm{v} \in \mathrm{I}} \mathrm{R}\left(\mathrm{l}_{\mathrm{v}}\right)+\sum_{(\mathrm{u}, \mathrm{v}) \mathrm{S} S} \mathrm{~V}\left(1_{u}, 1_{v}\right)
$$

where $1_{v}$ denotes the binary label assigned to the voxel $v, R\left(1_{v}\right)$ represents the region cost assigned to the voxel, $S$ is the $3 \mathrm{D}$ neighborhood system, (u, v) represent two neighboring voxels in the $3 \mathrm{D}$ neighborhood system, and $\mathrm{V}\left(\mathrm{l}_{\mathrm{u}}, \mathrm{l}_{\mathrm{v}}\right)$ is the corresponding boundary cost.

Region cost represents the likelihood of a voxel being assigned to the tumor or background. Generally speaking, to compute the region term, a seed set that requires user intervention is initialized at first. Subsequently, a learned intensity model, e.g., a histogram (25) or a Gaussian mixture model $(29,30)$, is used to build gray features for the region term. However, manual help initialization is inconvenient and the given intensity model may not fit the clinical MR data distribution in certain situations. To solve these problems, the probability response map, which is the output of the first stage of the proposed method, is used to obtain the region term $\mathrm{R}$. The probability response map is generated directly from the raw MR image without any prior assumptions on the data distribution, thus fitting the original image features. As the intensity values of the probability response map represent the likelihood of each voxel to be assigned to the tumor, the region cost may be expressed as follows:

$$
\begin{gathered}
\mathrm{R}_{\mathrm{v}}\left(\mathrm{l}_{\mathrm{v}}=1\right)=\lambda_{1}\left(1-\mathrm{i}_{\mathrm{v}}\right) \\
\mathrm{R}_{\mathrm{v}}\left(1_{\mathrm{v}}=0\right)=\lambda_{2} \mathrm{i}_{\mathrm{v}}
\end{gathered}
$$

where $1_{v}=1$ means that the voxel belongs to the tumor, $1_{v}=0$ means that the voxel is considered to be the background, $i_{v}$ denotes the intensity value for voxel $\mathrm{v}$ of the probability response map, and $\lambda_{1}$ and $\lambda_{2}$ are the two given scaling constants.

The boundary cost is designed to measure the difference between the two voxels in the 3D neighborhood and encourages two similar neighboring voxels to be assigned the same label. Thus, $\mathrm{V}\left(1_{\mathrm{u}}, 1_{\mathrm{v}}\right)$ may be expressed as follows:

$$
\mathrm{V}\left(\mathrm{l}_{\mathrm{u}}, \mathrm{I}_{\mathrm{v}}\right)=\left\{\begin{array}{l}
\mathrm{B}_{\mathrm{uv}}, \mathrm{l}_{\mathrm{u}} \neq \mathrm{I}_{\mathrm{v}} \\
0, \text { if } \mathrm{l}_{\mathrm{u}}=\mathrm{I}_{\mathrm{v}}
\end{array}\right\}
$$

where $\mathrm{B}_{\mathrm{uv}}$ is the penalty for assigning different labels to two neighboring voxels. Typically, $\mathrm{B}_{\mathrm{uv}}$ is defined as follows (22):

$$
\mathrm{B}_{\mathrm{uv}}=\lambda_{3} \exp \left[-\frac{|\nabla \mathrm{II}|^{2}(\mathrm{u}, \mathrm{v})}{2 \sigma^{2}}\right]
$$

where $|\nabla I|^{2}(u, v)$ represents the squared intensity gradient between the voxels $u$ and $v, \sigma$ is a given parameter associated with acquisition noise and $\lambda_{3}$ is a given scaling constant.

After region and boundary terms are computed, the energy minimization problem is transformed into a minimum s-t cut on the constructed 3D graph. The minimum s-t cut, which may be obtained by solving a maximum flow problem in low-order polynomial time, separates the $3 \mathrm{D}$ graph into two parts, the source set $\mathrm{A}$ and the sink set $\overline{\mathrm{A}}$ with $\mathrm{s} \in \mathrm{A}, \mathrm{t} \in \overline{\mathrm{A}}$ and $\mathrm{A} \cup \overline{\mathrm{A}}=\mathrm{N} \cup\{\mathrm{s}, \mathrm{t}\}$. The target $\mathrm{NPC}$ volume in the MR image is defined by those voxels whose corresponding nodes in the $3 \mathrm{D}$ graph belong to the source set.

Since the region cost is derived from the multi-view CNN method without any user intervention and the boundary term is designed to enforce the smooth structure, a fully-automated method was achieved by utilizing the $3 \mathrm{D}$ context information and the $3 \mathrm{D}$ structure information.

Extraction of patches. The method established above was then experimentally verified. Information on the classification of each voxel was provided in the form of image patches, where the target voxel is in the center, and the single-view $\mathrm{CNN}$ was trained separately using image patches extracted from a specified orthogonal view of the T1W images and the corresponding labels. For positive patch extraction, tumor voxels were first randomly selected referring to the manual GT images and $\mathrm{m} x \mathrm{~m}$ specified view patches (centered at that voxel) were then extracted. For negative patches extraction, in view of the fact that segmentation performance is usually affected by boundary accuracy, the centered non-tumor voxels were constrained to be selected near the tumor boundary and the same-size patches as positive patches were extracted as negative patches. In the present study, $m=31$ was used, which was experimentally determined. Fig. 6 presents examples of positive and negative patches extracted from an axial view.

For the nasopharyngeal tumor dataset, a leave-one-subject-out cross validation strategy was used to evaluate the segmentation performance. This means that for each single-view CNN, the training and testing cycle was repeated 30 times. In each iteration, 29 of the 30 NPC images formed the source of training patches and the remaining image was the source of test samples. From each training image, 10,000 positive training patches and an equal number of negative training patches were randomly sampled, such that the training set comprised $\sim 580,000$ patches. At the testing stage, all corresponding view patches were extracted 
Table I. Convolutional neural network architecture parameters.

\begin{tabular}{lc}
\hline Stage/parameter & Value \\
\hline Initialization & \\
Weights & Xavier \\
Bias & 0 \\
Training & \\
Initial learning rate & 0.001 \\
Learning rate decay policy & Inverse decay \\
Gamma & 0.0002 \\
Power & 0.75 \\
Weight decay & 0.0005 \\
Momentum & 0.9 \\
Batch size & 100 \\
Epochs & 10 \\
\hline
\end{tabular}

from each testing image to increase the accuracy of the assessment.

Implementation details and parameter setting. In the present experiments, the CNN-based classification was implemented in $\mathrm{C}++$ using the Caffe package (31). To train the network, the cost function was minimized using a gradient-based optimization algorithm (9) and the partial derivatives were computed using the back-propagation algorithm. Xavier initialization (32) was used to avoid vanishing back-propagated gradients and Nesterov Momentum (33) was employed to accelerate the optimization algorithm. In addition, weight decay was used to regularize the classification and mini-batch learning was used to train the network. The detailed parameters are displayed in Table I.

The 3D graph cut-based segmentation method used in the present study was implemented in $\mathrm{C}++$. The parameter setting was empirically employed for all analyzed NPC subjects, with the coefficients $\lambda_{1}=\lambda_{2}=1$ set for the region term, and $\lambda_{3}=5$ and $\sigma=0.5$ for the boundary term.

Evaluation measures. The evaluation of the segmentation performance in the present study considered four metrics: Average symmetric surface distance (ASSD), Dice similarity coefficient (DSC), percent match (PM) and correspondence ratio $(\mathrm{CR})$. The ASSD measures the boundary surface distance error between the manual GT and the automated segmentation result, defined as follows:

$$
\mathrm{ASSD}=\frac{1}{2}\left[\frac{\sum_{\mathrm{mcM}_{4}} \min _{\mathrm{acA}_{4}} \mathrm{~d}(\mathrm{~m}, \mathrm{a})}{\left|\mathrm{M}_{\mathrm{s}}\right|}+\frac{\sum_{\mathrm{acA}_{\mathrm{s}}} \min _{\mathrm{meM}_{\mathrm{s}}} \mathrm{d}(\mathrm{a}, \mathrm{m})}{\left|\mathrm{A}_{\mathrm{s}}\right|}\right]
$$

where $M_{s}$ and $A_{s}$ denote the surface voxels of the manual and the automated segmentation, respectively, and $\mathrm{d}(\mathrm{a}, \mathrm{m})$ represents the Euclidean distance between a and $\mathrm{m}$. The DSC is used for volumetric error measurement and is calculated as $\mathrm{DSC}=2 \mathrm{TP} /(\mathrm{FN}+2 \mathrm{TP}+\mathrm{FP})$, where $\mathrm{TP}, \mathrm{FN}$ and $\mathrm{FP}$ are the number of true positive, false negative and false positive voxels, respectively. $\mathrm{PM}$ is the ratio of TP to the number of tumor voxels in the manual GT, and is defined as PM=TPx100/GT.CR measures the correspondence of the segmented result and the
Table II. Summary of quantitative evaluation for segmentation methods: naive graph-cut, multi-view CNN and proposed combined method based on the evaluation metrics DSC and ASSD.

\begin{tabular}{lcc}
\hline Method & DSC & ASSD (mm) \\
\hline Naive graph cut & $0.580 \pm 0.108$ & $2.980 \pm 0.574$ \\
Multi-view CNN & $0.838 \pm 0.034$ & $1.203 \pm 0.346$ \\
Proposed combined method & $0.851 \pm 0.031$ & $0.979 \pm 0.270$ \\
\hline
\end{tabular}

Values are expressed as the mean \pm standard deviation. CNN, convolutional neural network; ASSD, average symmetric surface distance; DSC, dice similarity coefficient.

manual GT by weighting the importance of TP and FP, and is calculated as $\mathrm{CR}=(\mathrm{TP}-0.5 \mathrm{FP}) / \mathrm{GT}$.

\section{Results and discussion}

Study of multi-view CNN classification. To demonstrate the effectiveness of the proposed multi-view CNN method, the performance achieved by the sagittal, coronal, and axial view $\mathrm{CNN}$ on all subjects was evaluated using leave-one-subject-out cross validation. Each single-view CNN was separately trained with the patches of the same size and other parameters used in the CNN architecture. All initial segmentation results were post-processed with the same morphological method. The segmentation performance achieved by the single-view CNNs and the multi-view CNN method are presented in Fig. 7 using box plots. The results indicate that the proposed multi-view CNN method outperformed each single-view method for segmenting NPC. Specifically, the proposed multi-view CNN method achieved a mean DSC of 0.838 . By contrast, the coronal, sagittal and axial view CNN achieved a mean DSC of $0.757,0.786$ and 0.828 , respectively. In terms of ASSD, the proposed multi-view CNN method gained a mean value of 1.203 , while the single view $\mathrm{CNN}$ provided mean values of 1.858, 1.762 and 1.361, respectively. For the PM and $\mathrm{CR}$, the proposed multi-view CNN method also achieved the highest mean value among these methods. Furthermore, as observed from Fig. 7, the proposed multi-view CNN method achieved the smallest variance. These data demonstrate that by utilizing the $3 \mathrm{D}$ context information, the proposed multi-view CNN method is effective in improving the segmentation performance and is more stable and reliable than single view methods. In addition, the axial view CNN had the best performance among the three single-view CNNs. This may be explained by the acquisition of the axial view having a higher spatial resolution than the other two views and providing more useful information for discriminating the tumors in NPC subjects.

Validation of the proposed method. Experiments were performed to compare the performance of the naive 3D graph cut-based method, the proposed multi-view CNN approach alone and the proposed combined method. These three methods were applied to all subjects with the same pre-processing. The 
A
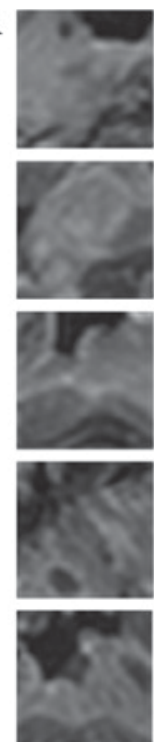
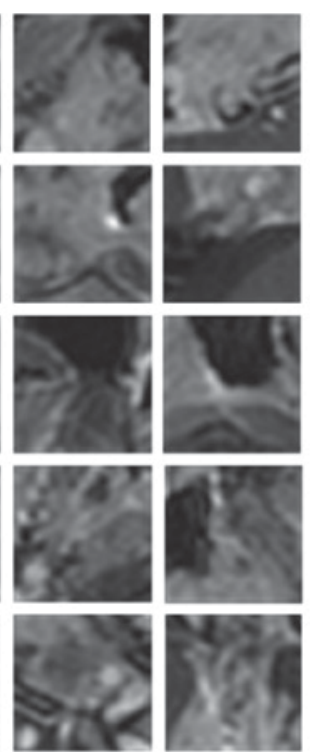
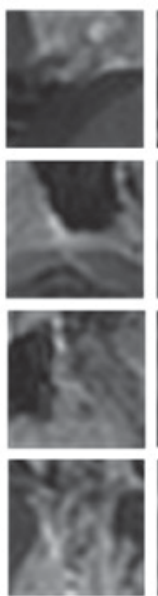
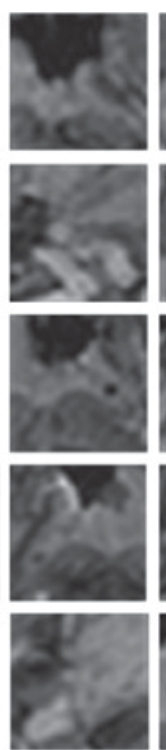
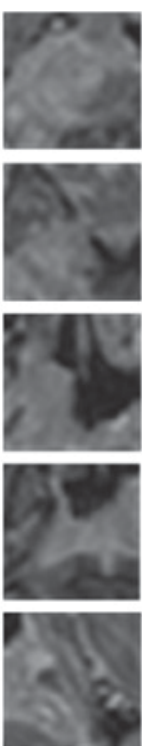
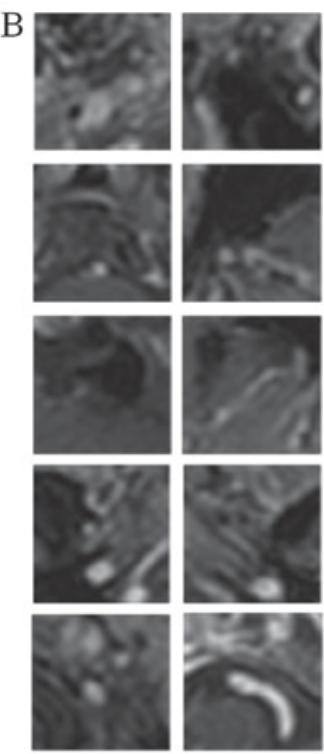
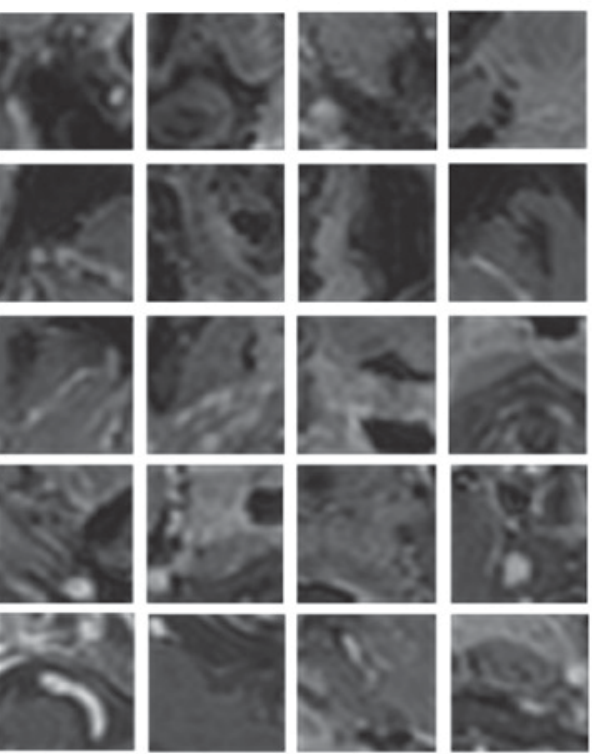

Figure 6. Examples of (A) positive and (B) negative training patches extracted from axial view of the training magnetic resonance images.
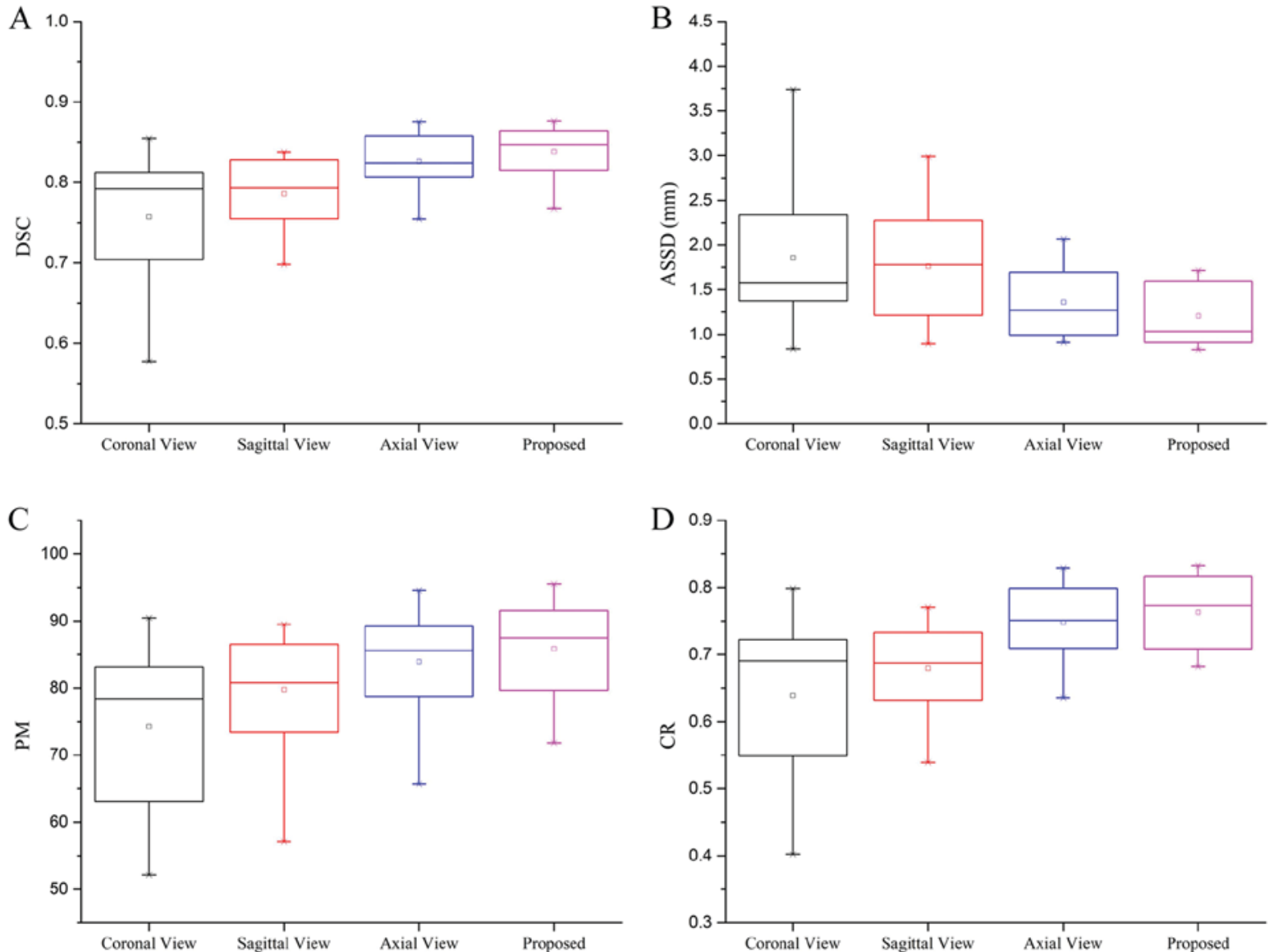

Figure 7. Comparison of segmentation performance of the single-view CNNs and the proposed multi-view CNN method in terms of (A) DSC, (B) ASSD, (C) PM and (D) CR. The top of the rectangle of the box plot indicates the third quartile, the bottom of the rectangle indicates the first quartile and the middle of the rectangle indicates the median. The vertical line extended from the top of the rectangle indicates the maximum value and the vertical line extended from the bottom of the rectangle indicates the minimum value. CNN, convolutional neural network; ASSD, average symmetric surface distance; DSC, dice similarity coefficient; PM, percent match; CR, correspondence ratio.

naive 3D graph cut-based method refers to the utilization of the same graph construction procedure, boundary cost formula and coefficients as the $3 \mathrm{D}$ graph cut-based segmentation described in materials and methods section, while the region cost was assigned according to the intensity distribution learned from the manual initialized seed set as reported previously (30). 

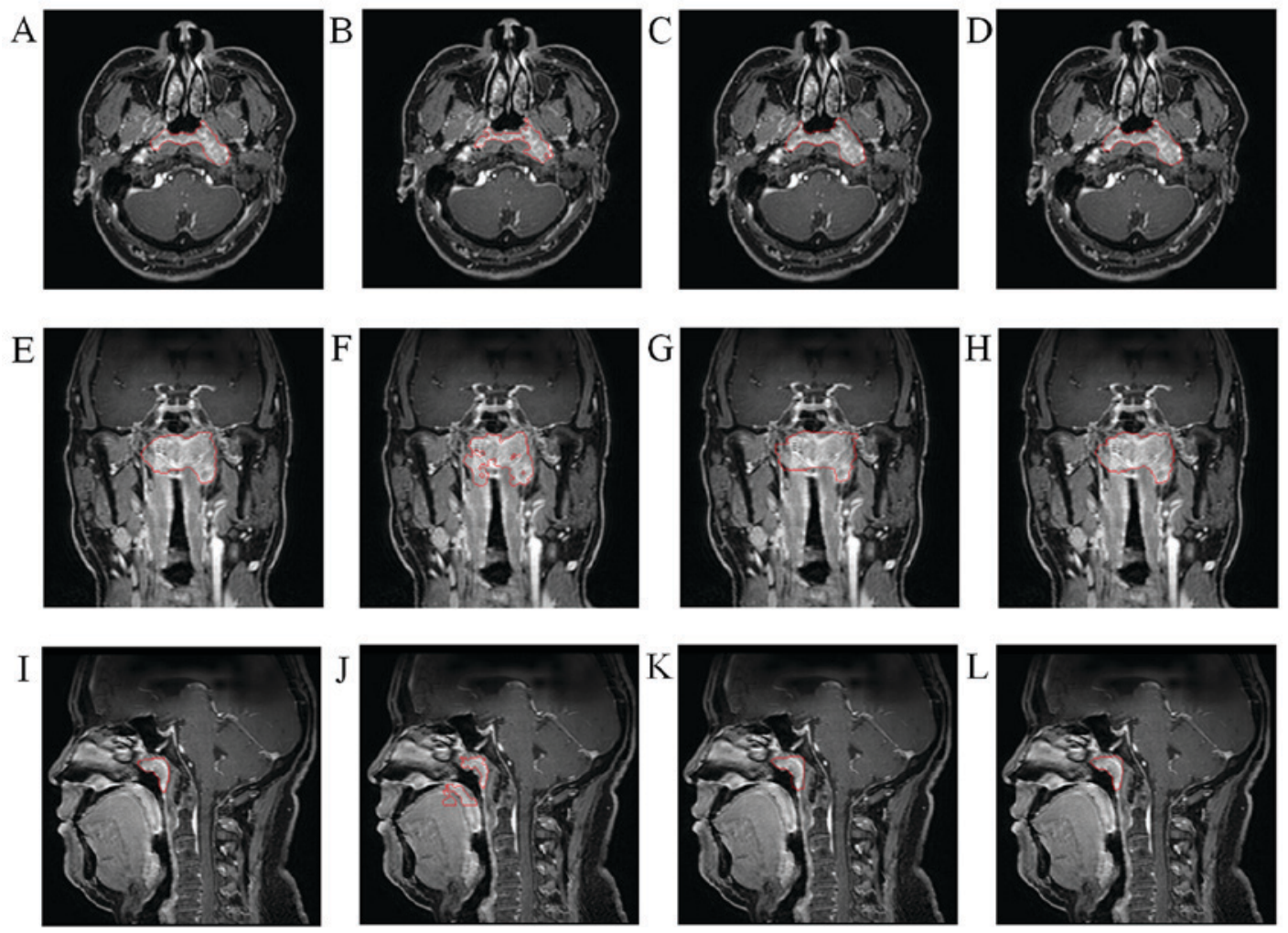

Figure 8. Typical tumor segmentation in the (A-D) axial, (E-H) coronal and (I-L) sagittal views. The first column displays the results obtained with manual segmentation, the second column those obtained with the naive 3-dimensional graph cut-based method and the third and fourth columns those generated with the multi-view convolutional neural network method and the proposed combined method, respectively.

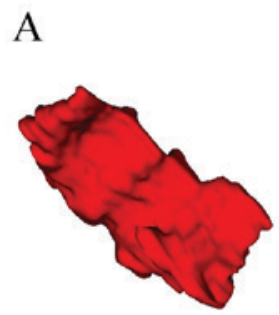

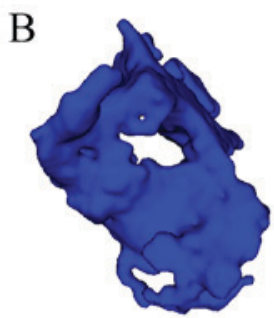

$\mathrm{C}$

F

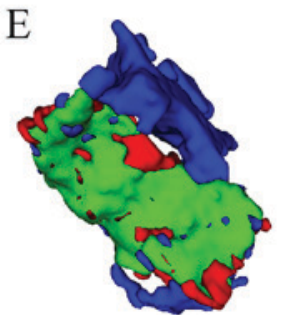

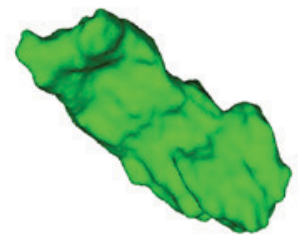

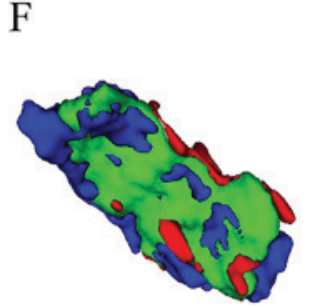

$\mathrm{D}$

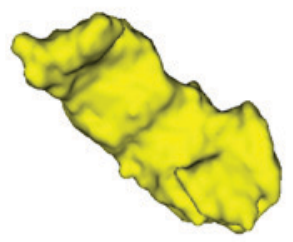

G

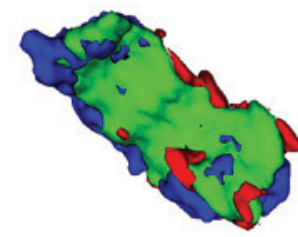

Figure 9. 3D visualization of the segmented nasopharyngeal carcinoma results obtained with different methods. (A-D) Results obtained with the (A) ground truth, (B) naive 3D graph cut-based method, (C) multi-view convolutional neural network-based segmentation and (D) the proposed combined method. (E-G) Overlay of ground truth and a corresponding segmentation method (red, ground truth; blue, corresponding segmentation method, naive 3D graph cut-based method in E, multi-view convolutional neural network-based segmentation in F and the proposed combined method in G; green, overlapped region between manual segmentation and a corresponding segmentation method). 3D, 3-dimensional.

The qualitative results from three views for a randomly selected subject of the present study are presented in Fig. 8. It may be observed that the multi-view CNN method achieved better results than the naive $3 \mathrm{D}$ graph cut and that the proposed combined method further improved the border quality of the multi-view CNN method. A similar trend is indicated in the 3D mesh overlay results of the GT and the corresponding segmentation methods presented in Fig. 9. Furthermore, Fig. 9 clearly indicates that the segmentation result achieved by the proposed combined method was closer to the GT than the other two methods.

Quantitative evaluation results in terms of the evaluation metrics DSC and ASSD are summarized in Table II. The results demonstrate that the proposed combined method achieved 
Table III. Comparison with two other previous segmentation methods for nasopharyngeal carcinoma.

\begin{tabular}{lcll}
\hline Study & PM & CR & Type \\
\hline Huang et al (2015) & $85.65 \pm 10.70$ & $0.72 \pm 0.10$ & Automated \\
Zhou et al (2006) & $79.00 \pm 7.00$ & $0.72 \pm 0.06$ & Semi-automated \\
Present study & $85.93 \pm 8.17$ & $0.77 \pm 0.05$ & Automated \\
\hline
\end{tabular}

Values are expressed as the mean \pm standard deviation. PM, percent match; CR, correspondence ratio.
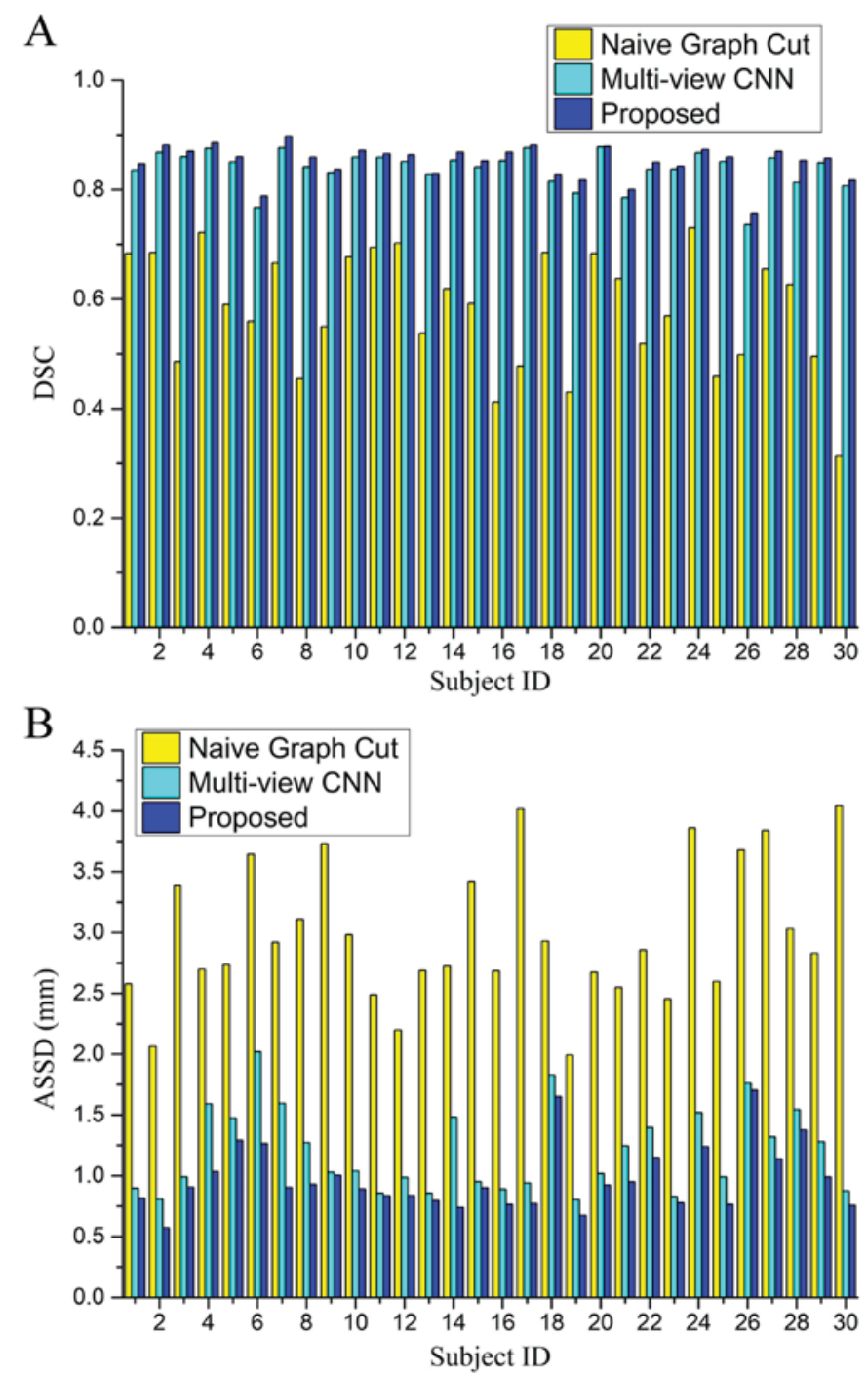

Figure 10. Quantitative and comparative performance evaluation based on computed (A) DSC values and (B) ASSD values for all nasopharyngeal carcinoma subjects. CNN, convolutional neural network; ASSD, average symmetric surface distance; DSC, dice similarity coefficient.

a significant improvement. Fig. 10 provides a comparison of the performance of the naive 3D graph cut based method, the proposed multi-view CNN alone approach and the proposed combined method for all NPC subjects. It may be observed that the proposed method is effective in all individual cases and has a consistent segmentation performance in the entire dataset.

The qualitative and quantitative evaluation results indicate that the segmentation performance of the naive 3D graph cut based method was less than satisfactory, which may have been due to the low intensity contrast between the tumor and its surrounding tissue. This suggests that the intensity-based method may not be suitable for the data used. For the proposed multi-view CNN approach, the segmentation performance significantly outperformed the naive 3D graph cut method. This is primarily because the CNN based method is able to automatically extract more complex features from the raw data and fully utilizes 3D context information via multi-view fusion. However, this fusion does not use a true 3D connection. Segmentation inferences for each individual voxel are independent of each 
other without considering structural information. Furthermore, it was observed that certain misclassified voxels were located in areas with large intensity contrast, which is clearly visible in Fig. 8. This may be due to the fact that the CNN method is patch-based, and patches centered at boundary voxels contain voxels with multiple classifications. In comparison, the proposed combined method, which was already initialized under near-optimal conditions by the multi-view CNN method to a certain extent, utilized 3D structural information and a global optimal solution provided by the graph cut to further refine the segmentation. This resulted in a more accurate segmentation, particularly in the tumor and non-tumor boundary regions.

Comparison with other NPC segmentation methods. Previously reported NPC segmentation methods $(5,7)$ were used to evaluate the proposed combined method and the comparison results are presented in Table III. PM and CR were selected as metrics to evaluate the segmentation performance, as they were used in these two previous methods. It should be noted that comparing these methods on different dataset may not be sufficiently objective, but the analysis gives an approximate estimation of the effectiveness of the method proposed by the present study. It was indicated that among the methods compared, the method of the present study obtained the highest mean value for PM and CR and achieved the lowest standard deviation in most cases, indicating that the present method is comparable to the previous methods.

In conclusion, the present study provided a novel fully-automated method for NPC segmentation of MR images. The proposed method combines a deep CNN model and a 3D graph cut method in a two-stage manner. First, a multi-view deep CNN-based segmentation method is performed, which directly learns features from data and fully utilizes 3D context information via multi-view fusion. In order to solve the true $3 \mathrm{D}$ structure and enforce smoothness, the multi-view CNN method was then combined into a graph cut framework. Since the multi-view CNN method provides initialization for the subsequent graph cut method, a fully-automated approach was achieved without any user intervention. The experimental results then verified that the present approach is effective and accurate for NPC segmentation.

\section{Acknowledgements}

The authors would like to thank Dr Shanhui Sun, CuraCloud Corporation (Seattle, WA, USA) for his valuable suggestions and proofreading of the manuscript. Part of the results published in the present study were previously presented at the 2017 International Conference on the Frontiers and Advances in Data Science on 23-25 October in China (19).

\section{Funding}

The present study was supported in part by the National Natural Science Foundation of China (grant no. NSFC61701324).

\section{Availability of data and materials}

The datasets used and/or analyzed during the current study are available from the corresponding author on reasonable request.

\section{Authors' contributions}

$\mathrm{ZM}, \mathrm{XW}, \mathrm{QS}$ and $\mathrm{JZ}$ conceived and designed the present study. ZM, XW and YL collected data and prepared the study. ZM and QS performed the experiments and wrote the manuscript. YW helped perform the analysis with constructive discussions. All authors have read and approved the final study.

\section{Ethical approval and consent to participate}

The present study was approved by the Ethics Committee of West China Hospital (Chengdu, China) and all patients gave written informed consent.

\section{Patient consent for publication}

Not applicable.

\section{Competing interests}

The authors declare that they have no competing interests.

\section{References}

1. Chang ET and Adami HO: The enigmatic epidemiology of nasopharyngeal carcinoma. Cancr Epidemiol Biomarkers Prev 15: 1765-1777, 2006.

2. Lee FK, Yeung DK, King AD, Leung SF and Ahuja A: Segmentation of nasopharyngeal carcinoma (NPC) lesions in MR images. Int J Radiat Oncol Biol Phys 61: 608-620, 2005.

3. Tatanun C, Ritthipravat $\mathrm{P}$, Bhongmakapat $\mathrm{T}$ and Tuntiyatorn $\mathrm{L}$ : Automatic segmentation of nasopharyngeal carcinoma from CT images: Region growing based technique. In Signal Processing Systems (ICSPS), 2010. 2nd International Conference on, 2010 (DOI: 10.1109/ICSPS.2010.5555663).

4. Chanapai W, Bhongmakapat T, Tuntiyatorn L and Ritthipravat P: Nasopharyngeal carcinoma segmentation using a region growing technique. Int J Comput Assist Radiol Surg 7: 413-422, 2012.

5. Huang KW, Zhao ZY, Gong Q, Zha J, Chen L and Yang R: Nasopharyngeal carcinoma segmentation via HMRF-EM with maximum entropy. Conf Proc IEEE Eng Med Biol Soc, pp2968-2972, 2015 (DOI: 10.1109/EMBC.2015.7319015).

6. Fitton I, Cornelissen SA, Duppen JC, Steenbakkers RJ, Peeters ST, Hoebers FJ, Kaanders JH, Nowak PJ, Rasch CR and van Herk M: Semi-automatic delineation using weighted CT-MRI registered images for radiotherapy of nasopharyngeal cancer. Med Phys 38: 4662-4666, 2011.

7. Zhou J, Chan KL, Xu P and Chong VFH: Nasopharyngeal carcinoma lesion segmentation from MR images by support vector machine. In Biomedical Imaging: Nano to Macro, 2006. The 3rd IEEE International Symposium on, 2006 (DOI: 10.1109/ISBI.2006.1625180).

8. Zhou J, Lim TK, Chong V and Huang J: Segmentation and visualization of nasopharyngeal carcinoma using MRI. Comput Biol Med 33: 407-424, 2003.

9. LeCun Y, Bottou L, Bengio Y and Haffner P: Gradient-based learning applied to document recognition. Proc IEEE 86: 2278-2324, 1998.

10. Krizhevsky A, Sutskever I and Hinton GE: ImageNet classification with deep convolutional neural networks. in Adv Neural Inf Process Syst 1, pp1097-1105, 2012, 2012.

11. Prasoon A, Petersen K, Igel C, Lauze F, Dam E and Nielsen M: Deep feature learning for knee cartilage segmentation using a triplanar convolutional neural network. Med Image Comput Comput Assist Interv 16: 246-253, 2013.

12. Roth HR, Farag A, Lu L, Turkbey EB and Summers RM: Deep convolutional networks for pancreas segmentation in CT imaging. SPIE Med Imag: 94131G, 2015 (DOI: 10.1117/12.2081420).

13. Liskowski P and Krawiec K: Segmenting retinal blood vessels with deep neural networks. IEEE Trans Med Imaging 35: 2369-2380, 2016. 
14. Zhang W, Li R, Deng H, Wang L, Lin W, Ji S and Shen D: Deep convolutional neural networks for multi-modality isointense infant brain image segmentation. Neuroimage 108: 214-224, 2015.

15. Moeskops P, Viergever MA, Mendrik AM, de Vries LS, Benders MJ and Isgum I: Automatic segmentation of MR brain images with a convolutional neural network. IEEE Trans Med Imaging 35: 1252-1261, 2016.

16. Pereira S, Pinto A, Alves V and Silva CA: Brain tumor segmentation using convolutional neural networks in MRI images. IEEE Trans Med Imaging 35: 1240-1251, 2016.

17. Zikic D, Ioannou Y, Brown M and Criminisi A: Segmentation of brain tumor tissues with convolutional neural networks. MICCAI Multi Brain Tumor Segment Challeng (BraTS) 2014: pp36-39, 2014.

18. Havaei M, Ddavy A, Warde-Farley D, Biard A, Courville A, Bengio Y, Pal C, Jodoin PM and Larochelle H: Brain tumor segmentation with deep neural networks. Med Image Anal 35: 18-31, 2017.

19. Ma ZQ, Wu X and Zhou JL: Automatic nasopharyngeal carcinoma segmentation in MR images with convolutional neural networks. in 2017 Int Conference Front Adv Data, pp147-150, 2017.

20. Tustison NJ, Avants BB, Cook PA, Zheng Y, Eqan A, Yushkevich PA and Gee JC: N4ITK: Improved N3 bias correction. IEEE Trans Med Imaging 29: 1310-1320, 2010.

21. Nyúl LG, Udupa JK and Zhang X: New variants of a method of MRI scale standardization. IEEE Trans Med Imaging 19: 143-150, 2000

22. Hinton GE, Srivastava N, Krizhevsky A, Sutskever I and Salakhutdinov RR: Improving neural networks by preventing co-adaptation of feature detectors. Neural Evolution Comput, 2012.

23. Jarrett K, Kavukcuogly K, Ranzato M and LeCun Y: What is the best multi-stage architecture for object recognition? IEEE: 2146-2153, 2009.
24. Boykov Y, Veksler O and Zabih R: Fast approximate energy minimization via graph cuts. IEEE Trans Pattern Anal Mach Intell 23: 1222-1239, 2001.

25. Boykov Y and Funka-Lea G: Graph cuts and efficient N-D image segmentation. Int J Comput Vis 70: pp109-131, 2006.

26. Grosgeorge D, Petitjean C, Dacher JN and Ruan S: Graph cut segmentation with a statistical shape model in cardiac MRI. Comput Vis Image Understand 117: 1027-1035, 2013.

27. Martinez-Muñoz S, Ruiz-Fernandez D, Galiana-Merino JJ: Automatic abdominal aortic aneurysm segmentation in MR images. Expert Syst Applicat 54: 78-87, 2016.

28. Mahapatra D and Buhmann JM: Prostate MRI segmentation using learned semantic knowledge and graph cuts. IEEE Trans Biomed Eng 61: 756-764, 2014.

29. Tian Z, Liu L, Zhang Z and Fei B: Superpixel-based segmentation for 3D prostate MR images. IEEE Trans Med Imaging 35: 791-801, 2016.

30. Song Q, Bai J, Han D, Bhatia S, Sun W, Rockey W, Bayouth JE, Buatti JM and Wu X: Optimal co-segmentation of tumor in PET-CT images with context information. IEEE Trans Med Imaging 32: 1685-1697, 2013.

31. Jia Y, Shelhamer E, Donahue J, Karayev S, Long J, Girshick R, Guadarrama S and Darrell T: Caffe: Convolutional architecture for fast feature embedding. In Proceed of the 22nd ACM Int Conferen Multimedia, ACM, pp675-678, 2014.

32. Glorot $X$ and Bengio $Y$ : Understanding the difficulty of training deep feedforward neural networks. in Proc Int Conf Artif Intell Stat 2010: pp249-256, 2010

33. Sutskever I, Martens J, Dahl G and Hinton G: On the importance of initialization and momentum in deep learning. PMLR 28: 1139-1147, 2013.

(i) (9) This work is licensed under a Creative Commons CC) Attribution-NonCommercial-NoDerivatives 4.0 International (CC BY-NC-ND 4.0) License. 